\section{6 OPEN ACCESS}

- Additional material is published online only. To view please visit the journal online (http://dx.doi.org/10.1136/ bjophthalmol-2013-303367).

Helen Keller Foundation for Research and Education, Birmingham, Alabama, USA

\section{Correspondence to} Robert E Morris, Helen Keller Foundation for Research and Education, 1201 11th Avenue South, Suite 300, Birmingham, AL 35205, USA

Received 4 March 2013 Revised 13 May 2013 Accepted 26 May 2013 Published Online First 21 June 2013

\title{
Presumed Air by Vitrectomy Embolisation (PAVE) a potentially fatal syndrome
}

\author{
Robert E Morris, Mathew R Sapp, Matthew H Oltmanns, Ferenc Kuhn
}

\begin{abstract}
Background Since first being reported in the ophthalmology literature in 2010, three cases (one fatal) of suspected venous air embolism (VAE) during vitrectomy have received little notice, and the vitrectomy/ VAE connection has been described as unproven. We investigated the ability of air to exit the eye through vortex veins after accidental suprachoroidal air infusion. Methods Vitrectomy was performed on four donor eyes. Unsutured cannulas were partially withdrawn during air fluid exchange, producing choroidal detachments that emulated accidental suprachoroidal air infusion from a slipping cannula. Eyes with and without clamping of the vortex vein stumps were partially submerged in a water bath.
\end{abstract}

Results Extensive choroidal detachment was created in all eyes during air infusion. All eyes with open vortex veins demonstrated rapid air extravasation/bubbling. An eye with clamped vortex vein stumps showed no air extravasation until the clamps were removed.

Conclusions When combined with existing clinical reports of suspected VAE in the eyes of living patients during ocular air fluid exchange, this experiment justifies recognition of presumed air by vitrectomy embolisation (PAVE) as a rare but potentially fatal vitrectomy complication. Simple surgical precautions can change PAVE from a 'rare event' to a 'never event', beginning with acknowledgment of its existence.

\section{INTRODUCTION}

A report in the anaesthesia literature in 2005 described a case of possible life-threatening venous air embolism (VAE) occurring concurrently with ocular air fluid exchange (OAFE) during vitrectomy. ${ }^{1}$ Lim, Somerville and Walker subsequently reviewed, in an ophthalmology journal in 2010, three suspected OAFE/VAE cases (one fatal) previously appearing only in anaesthesia journals, in order to finally bring these cases to the attention of ophthalmologists who could prevent VAE during vitrectomy.

Most vitreoretinal surgeons apparently remain unaware of the possibility that OAFE during vitrectomy could enable life-threatening VAE within minutes of air infusion initiation, despite these reported cases. ${ }^{2}$ Only 2 of 24 vitreoretinal surgeons we individually polled in the USA in May 2012 were aware of a potential OAFE/VAE connection, and an informal group discussion with European ophthalmologists yielded similar results. Most surgeons expressed surprise that the relatively small venous outflow of the eye could enable lifethreatening VAE.

Similarly, an August 2012 survey of 10 prominent specialty eye surgery facilities throughout the USA revealed that none had a policy defining infusion procedures for surgical teams performing OAFE during vitrectomy. This adequately demonstrates that, 2 years after Lim's warning, and 7 years after the first VAE report, there is still no standard of care in the vitreoretinal community to prevent VAE during vitrectomy eye surgery.

Lim's article was met by only one letter of comment that characterised the OAFE connection to VAE as still unproven despite the reported cases. ${ }^{3}$ The aims of our article are to provide additional evidence that inadvertent suprachoroidal air infusion could tear vortex veins and rapidly entrain air into the systemic circulation, as described by Ledowski and $\operatorname{Lim}^{1} 2$ (figure 1); to show that such an occurrence may be more likely, as vitrectomy has increasingly moved to 25-guage and 23-gauge unsutured infusion cannulas; and to point out that, once acknowledged, the risk of fatal VAE during OAFE can be almost eliminated by simple procedural precautions. These procedures could then establish an initial standard of care for infusion and VAE prevention during vitrectomy.

\section{MATERIALS AND METHODS}

To simulate accidental suprachoroidal air infusion, a banked enucleated eye unsuitable for tissue donation was first placed in a gauze-filled orbit of a mannequin head. The central opacified cornea was removed with an $8 \mathrm{~mm}$ trephine. The lens was removed, and a plastic cornea (temporary keratoprosthesis) was emplaced to provide excellent visualisation of the vitreous cavity. Vitrectomy was performed through trocar-inserted cannulas.

The infusion cannula was then slowly backed out to simulate accidental cannula slippage, and OAFE was begun at $30 \mathrm{~mm} \mathrm{Hg}$. Air entered the vitreous cavity and a choroidal detachment quickly ensued, obscuring vitreous cavity visualisation. The eye was then transported to an adjacent water bath. It was dipped to its equator so that the vortex vein stumps were submerged while all surgical incisions were 'high and dry'. This experiment was performed in three eyes, using both 25-guage and 23-gauge systems, and under intraocular pressures varying between $30 \mathrm{~mm} \mathrm{Hg}$ and $60 \mathrm{~mm} \mathrm{Hg}$.

In a fourth eye, the four vortex vein stumps (figure 2) were closed with serafin clamps before submersion, and the clamps were removed before resubmersion, to investigate the possibility that air had exited through either the optic nerve sheath or the (vortex vein) scleral canals, rather than through the vortex vein lumina.

\section{RESULTS}

After production of a choroidal detachment by air infusion and transportation to an adjacent water 


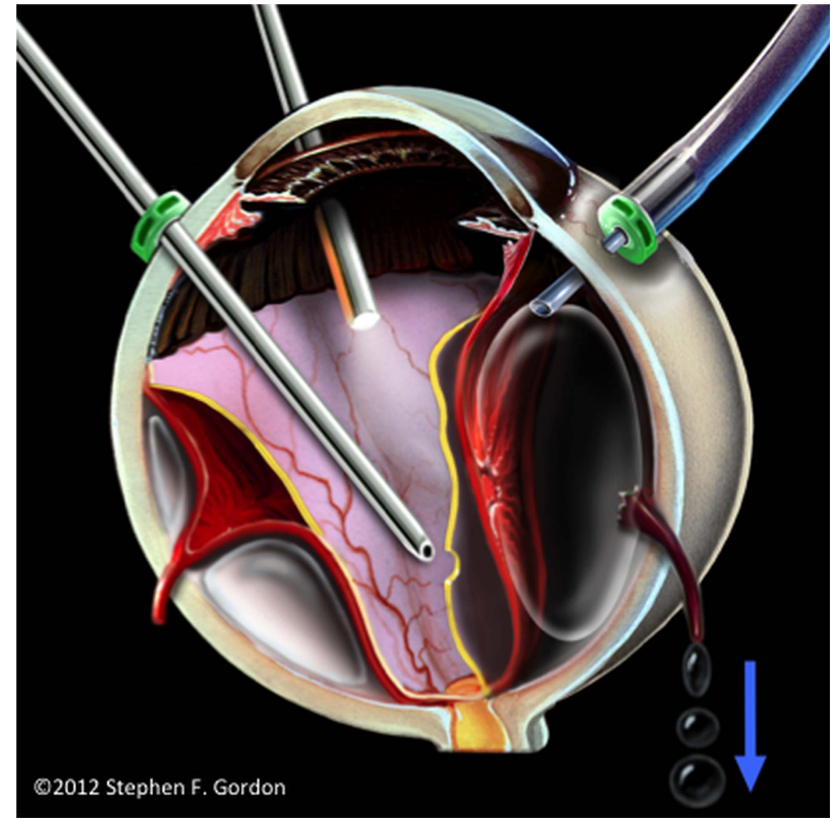

Figure 1 Concept illustration of pressurised air from a slipping, unsecured cannula causing accidental choroidal detachment, torn vortex vein ampullae and air entrainment through vortex veins.

bath, air bubbled upward profusely from the submerged, postequatorial sclera of the first three eyes, at a rate proportional to the intraocular pressure being used. In the fourth eye, no air exited through the water bath until the serafin clamps were removed from the vortex veins, at which point air again bubbled profusely, as shown in the supplementary video.

\section{DISCUSSION}

Two of the three cases of suspected OAFE/VAE thus far reported in the medical literature involved abnormal venous access through wounds caused by intraocular foreign body trauma. ${ }^{2}$ Such wounds are rare, but they must now be considered as a

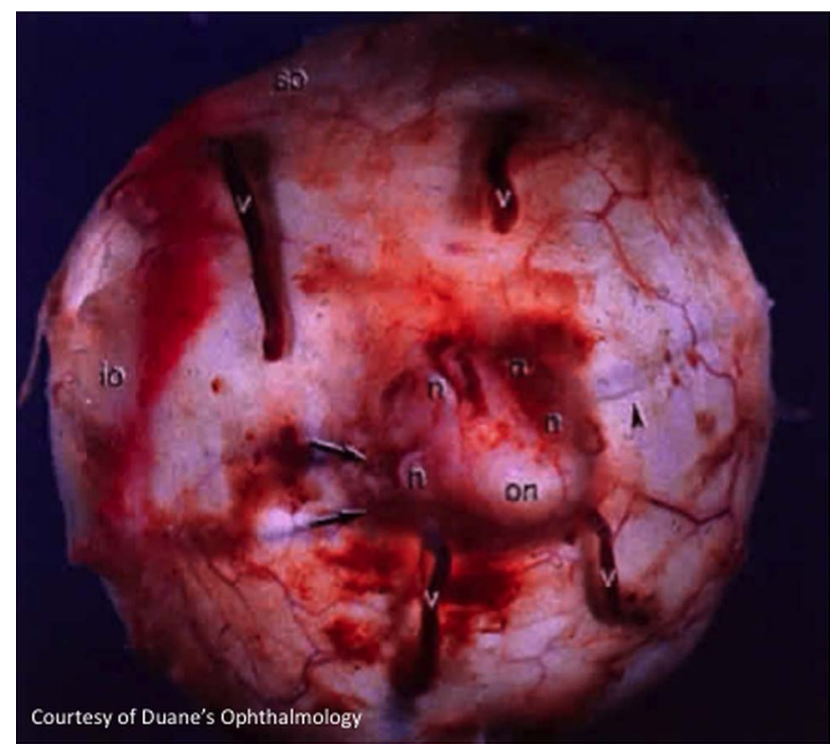

Figure 2 Vortex veins exiting the posterior aspect of an enucleated eye. (Courtesy of Duane's Ophthalmology, Lippincott Williams \& Wilkins). forewarning of possible VAE entering through the eye during vitrectomy for such posterior segment trauma.

More insidious, and of even greater concern, is the possible creation of choroidal detachment by inadvertent suprachoroidal air infusion during vitrectomy performed for more common conditions, such as macular hole and retinal detachment. ${ }^{12}$ Air under continuous pressure might then enter the systemic venous circulation through torn vortex vein ampullae ${ }^{123}$ (figure 1), at a rate sufficient to quickly block pumping action of the heart, ${ }^{4}$ or to cause fatal emboli into the arterial circulation through a patent foramen ovale. ${ }^{2}$ In fact, using $40 \mathrm{~mm} \mathrm{Hg}$ infusion pressure through a 25 -gauge line, we measured air flow against atmospheric pressure to be approximately $350 \mathrm{ml}$ per minute. Since an embolism as small as $200 \mathrm{ml}$ can be fatal, ${ }^{4}$ a vitreoretinal surgeon might have only seconds to minutes to effectively stop air infusion after choroidal elevation appears as a first warning sign, depending on the infusion gauge in use, the extent of vortex vein tearing, and the infusion pressure selected before and after choroidal detachment is detected.

While such an occurrence may initially seem implausible, it is not so difficult to imagine upon reflection. Each of the ocular vortex veins can be as large as the infusion cannula (figures 2 and 3), and entrainment through these vortex veins is the most likely explanation for CT imaged air in the orbit in the report of possible OAFE/VAE by Ledowski. ${ }^{1}$ Moreover, a choroidal detachment was actually seen during the OAFE in Ledowski's report, and the characteristic mill-wheel murmur of VAE stopped immediately after ocular air infusion was discontinued, with prompt cardiopulmonary recovery.

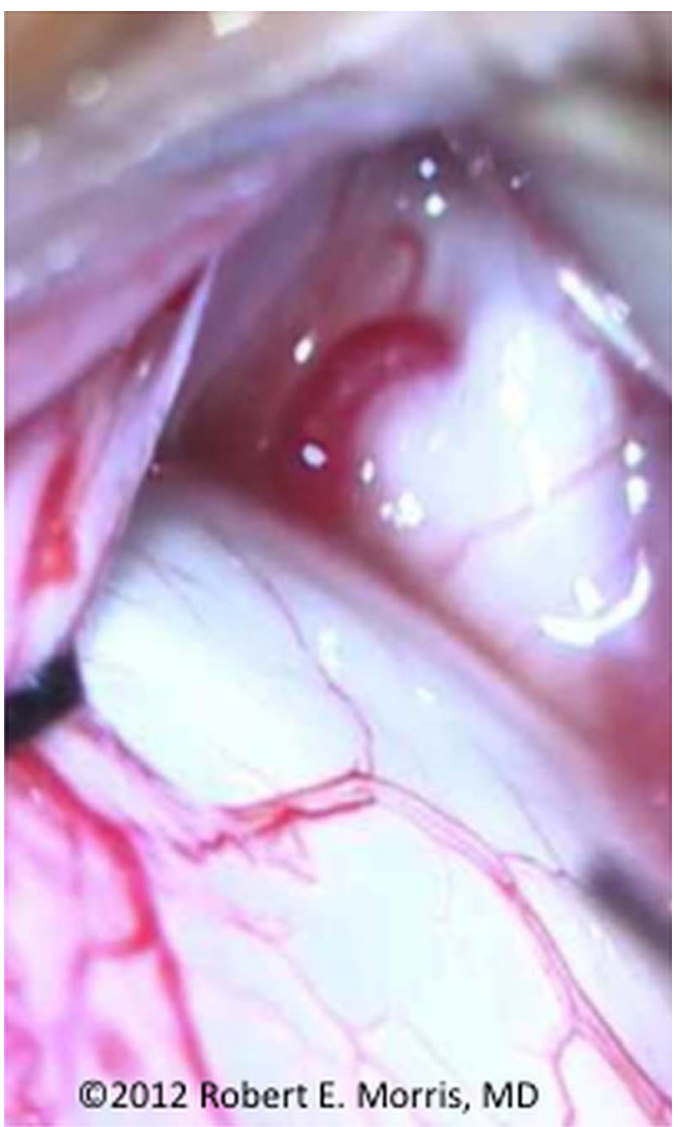

Figure 3 Vortex vein, after conjunctiva retracted intraoperatively, exiting posterior scleral eye wall. 
Our corroborating experiment in enucleated eyes, while of clear importance as visual evidence, is admittedly not performed in conditions identical to those seen in the eye of a living patient, but they are reasonably close, and when analysed in conjunction with the reports that inspired it, ${ }^{12}$ our experiment is compelling evidence for an OAFE/VAE connection. Air passage occurred only through vortex vein lumina in our experiment, and we believe such passage only could account for Ledowski's suspected VAE observations in a living patient during OAFE.

We think this experiment, when considered together with the clinical reports of suspected VAE, constitutes proof beyond a reasonable doubt that OAFE is capable of causing fatal VAE through torn vortex veins during inadvertent suprachoroidal air infusion because no other logical explanation can be derived from the facts of these reports and this experiment.

The infusion cannula in our experiment was trocar inserted through intact conjunctiva, with no suturing to the sclera, because most vitrectomies are now so performed. The advantages of such small-gauge, sutureless vitrectomy ensure its continued predominance, but unsutured infusion cannulas often slip outward, requiring monitoring and repositioning, especially during prolonged vitrectomy with extensive eye movement (figure 4). As an infusion cannula slips outward, it can also swivel to a non-radial orientation, potentially engaging relatively large wounds in the choroid that are unseen when produced by trocars making bevelled sclerotomies. The eye could then be injured by accidental suprachoroidal infusion. Should this occur, inadvertent liquid infusion is curable by external drainage, or it may resolve spontaneously. However, if air infused suprachoroidally can cause potentially fatal VAE, we must respond decisively with new safety precautions. ${ }^{1-3}$

\section{PREVENTION OF VAE}

Many vitreoretinal surgeons have never seen a case of accidental suprachoroidal infusion, and for them it may be only a theoretical consideration. Even for those who have, scepticism that a fatal dose of suprachoroidal air could rapidly pass through the eye into the heart is understandable. ${ }^{3}$ The OAFE/VAE connection has only recently and rarely been reported, and VAE is typically a transitory, clinical diagnosis.

But VAE risk is likely higher now than in previous decades, when infusion cannulas were sutured to the sclera; and Albin noted that many cases of VAE are probably never published. ${ }^{5}$ For example, the three reported eye cases took years to enter

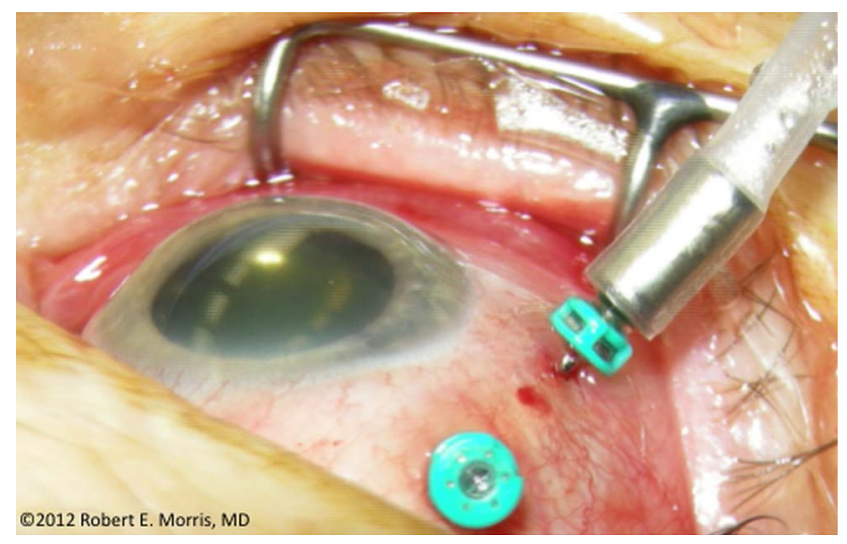

Figure 4 Intraoperative image of an unsutured infusion cannula insidiously slipping outward during vitrectomy. the ophthalmology literature, finally appearing only because an uninvolved ophthalmologist accidentally happened upon them while reading the anaesthesia literature for other reasons. ${ }^{2} 3$ These factors do not support OAFE/VAE scepticism. Furthermore, although the diagnosis of VAE depends on a constellation of temporary, clinical findings (precipitous drop in end-tidal carbon dioxide, falling peripheral oxygen saturation, haemodynamic collapse, mill-wheel murmur and profound cyanosis of the head and thorax), together they are pathognomonic, in the absence of another identifiable cause of cardiopulmonary collapse, during surgery that involves air infusion.

Since large and rapid air entrainment can be fatal despite expert resuscitative treatment, the goal of prevention is critically important. So how should vitreoretinal surgeons respond in the face of these disturbing VAE reports? Even in the absence of absolute proof, preventive efforts should start with unequivocal recognition of the potentially lethal possibility of a cause and effect relationship between OAFE and VAE.

We recommend use of the acronym Presumed Air by Vitrectomy Embolisation (PAVE) to encourage immediate recognition of concurrent OAFE and VAE as a syndrome (a set of things that form an identifiable pattern), and to promote simple VAE preventive efforts (box 1). Institutional policy should now require reconfirmation of infusion cannula position before beginning OAFE, and immediate discontinuance of air infusion if choroidal elevation is detected. To safeguard a patient's life, this is not too burdensome, anymore than a mandatory 'timeout' (as practiced in the USA) now effectively prevents wrong site surgery. Other preventive suggestions are offered for consideration in the accompanying box, and equipment/supply vendors could also make design changes to help this effort.

\section{CONCLUSION}

We cannot escape the unfortunate reality that the potential for fatal air embolism in eye surgery does exist; and that preventive

Box 1 Presumed Air by Vitrectomy Embolisation (PAVE) prevention

\section{Critical}

- Reconfirmation of infusion cannula immediately prior to OAFE.

- If choroidal elevation occurs during OAFE, air infusion is immediately stopped and the source of choroidal elevation is investigated.

\section{Advisory}

- Staff and physician education regarding PAVE.

- Prospective plan for venous air embolism management.

- Ensure that end-tidal CO2 (ETCO2) monitor is functioning. Consider sutured infusion and precordial Doppler monitor when prolonged surgery under air infusion is planned. ${ }^{4}$

- Confirmation of infusion before vitrectomy is initiated.

- Surgeon announces ocular air fluid exchange (OAFE) to anaesthesia personnel who acknowledge.

- Maximum air infusion pressure of $30 \mathrm{~mm} \mathrm{Hg}$ until air is seen directly entering the vitreous cavity.

- Lights-on surgical technician confirmation of 'Air Arriving' at the eye.

- Surgeon immediately confirms 'Air in Vitreous Cavity', and then proceeds to lights-out OAFE at desired pressure. 'Air Off' if air does not immediately enter the vitreous cavity. 
policies have not been established since the initial PAVE report in $2005 .{ }^{1}$ We had best not further delay an effective response until PAVE becomes even more tragically apparent, at the expense of even one more human life, when prevention is so easily within our power upon admission.

Preventing this potentially fatal complication should now become a fundamental concern of all vitrectomy surgeons and their host institutions. By eliminating inadvertent suprachoroidal air infusion, PAVE can be changed from a rare event to a 'never' event. In this preventive effort, guarding life while saving sight trumps scepticism and all other concerns.

Acknowledgements The authors gratefully acknowledge Ms Margaret Harrill and Mrs Christina Sullivan for their technical and clerical support; Mr Tom Cattell for video editing; the Alabama Eye Bank for providing donated eyes; Ms Stephanie Hill and Ms Tracy Morris for surgical assistance; Mr Stephen Gordon for medical illustration; and Professor Thomas Ledowski, MD, Maurice S Albin, MD, MSc (Anaes) and LT Lim, MD for their expert review.

Contributors All authors contributed to the planning and design of the study and assisted in the editing of the final manuscript.
Competing interests None.

Provenance and peer review Not commissioned; externally peer reviewed.

Open Access This is an Open Access article distributed in accordance with the Creative Commons Attribution Non Commercial (CC BY-NC 3.0) license, which permits others to distribute, remix, adapt, build upon this work non-commercially, and license their derivative works on different terms, provided the original work is properly cited and the use is non-commercial. See: http://creativecommons.org/ licenses/by-nc/3.0/

\section{REFERENCES}

1 Ledowski T, Kiese $\mathrm{F}$, Jeglin $\mathrm{S}$, et al. Possible air embolism during eye surgery. Anesth Analg 2005;100:1651-2.

2 Lim LT, Somerville GM, Walker JD. Venous air embolism during air/fluid exchange: a potentially fatal complication. Arch Ophthalmol 2010;128:1618-19.

3 El-Annan J, Barr CC. Does venous air embolism occur during air/fluid exchange? Arch Ophthalmol 2011;128:1244; author reply 1244-5.

4 Mirski M, Abjihit VL, Fitzsimmons L, et al. Diagnosis and treatment of vascular air embolism. Anesthesiology 2007;106:164-77.

5 Albin MS, Ritter RR, Sload T, et al. Central registry for venous air embolism. Anesth Analg 1995;81:658. 